\title{
Blancos terapéuticos potenciales para revertir la cirrosis hepática
}

\author{
Leonel G arcía B, Javier Gálvez G, Juan Armendáriz B. \\ Therapeutical targets \\ for revert liver fibrosis
}

Liver fibrosis is the common response to chronic liver injury, ultimately leading to cirrhosis and its complications: portal hypertension, liver failure, hepatic encephalopathy, and hepatocellular carcinoma and others. Efficient and well-tolerated antifibrotic drugs are still lacking, and current treatment of hepatic fibrosis is limited to withdrawal of the noxious agent. Efforts over the past decade have mainly focused on fibrogenic cells generating the scarring response, although promising data on inhibition of parenchymal injury or reduction of liver inflammation have also been obtained. A large number of approaches have been validated in culture studies and in animal models, and several clinical trials are underway or anticipated for a growing number of molecules. This review highlight recent advances in the molecular mechanisms of liver fibrosis and discusses mechanistically based strategies that have recently emerged (Rev Méd Chile 2007; 135: 783-91).

(Key words: Fatty liver; Fibrosis; Liver cirrhosis).

Recibido el 16 de marzo, 2006. Aceptado el 10 de octubre, 2006.

Instituto de Biología Molecular en Medicina y Terapia Génica, Departamento de Biología Molecular y Genómica, CUCS, Universidad de Guadalajara, México.

$E_{h}^{1}$ daño hepático crónico induce fibrosis en el hígado y en sus estados finales cirrosis, que se presenta como uno de los mayores problemas de salud pública a nivel mundial. La cirrosis hepática es una enfermedad que se caracteriza por una distorsión irreversible de la arquitectura hepática y una exacerbada acumulación de fibrosis. Los eventos moleculares encontrados en la cirrosis hepática implican: acúmulo de laminina $\alpha-1$, tenascina y fibronectina, con la sucesiva activación y proliferación de células estelares hepáticas

Correspondencia a: Dr. Francisco Javier Gálvez Gastélum. Sierra Mojada 950, puerta 7, Col. Independencia, Apdo. Postal 2-123, Tel/Fax (0155) 33 10585317. E mail: galvez_gastelum_@hotmail.com
(HSC) inducida mediante la secreción autocrina y paracrina de citocinas tales como: el factor de crecimiento derivado de plaquetas (PDGF) y el factor de crecimiento transformante $\beta$ tipo 1 (TGFß1) (Figura 1). Las HSC activadas (HSCa) conducen a un incremento en los depósitos de proteínas de matriz extracelular (MEC), fundamentalmente colágenas tipo I, III y IV en el espacio perisinusoidal o «de Disse», lo que dificulta el intercambio de substancias entre el sinusoide y los hepatocitos (Figura 2) ${ }^{1-4}$. Las manifestaciones clínicas se atribuyen a la disfunción hepatocelular progresiva e hipertensión portal ${ }^{5}$. Conforme progresa la enfermedad, los pacientes cursan con episodios cada vez más frecuentes de sangrado de tubo digestivo y de encefalopatía, complicaciones graves que los llevan a la muerte. 
Figura 1. Principales eventos moleculares que conducen a la cimosis hepática y que deben ser comegidos para el tratamiento de la enfermedad. La activación de las HSC presentes en el espacio subendotelial (espacio de Disse) que inducen una exacerbada expresión de proteínas fibrilares (principalmente colágena tipo I, III y VI) y una disminución en la actividad de enzimas (conocidas como metaloproteasas) que degradan dichas proteínas colagénicas, ocasionan una alteración en el intercambio de sustancias entre el sinusoide y los hepatocitos para ser metabolizadas son sólo algunos de los mecanismos involucrados durante el desarmollo de la cirmosis en el hígado.
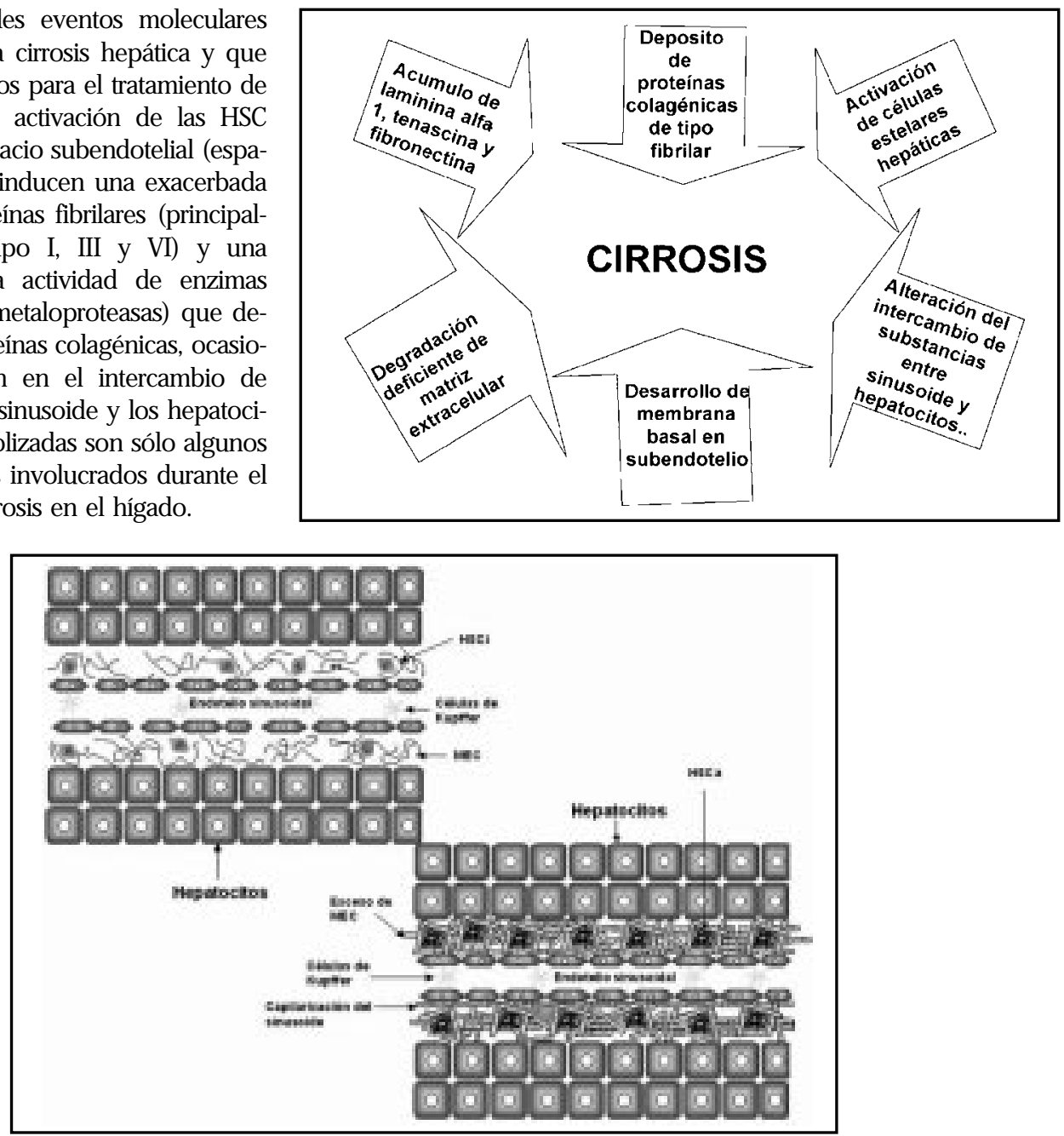

Figura 2. Alteraciones moleculares que conducen al desarrollo de la fibrogénesis hepática. Los inicios del proceso fibrogénico tienen lugar en el espacio subendotelial o espacio de Disse, en donde los actores principales son las células estelares hepáticas (HSC) que, tras un daño ocasionado por liberación de radicales libres (estrés oxidativo) entran en un proceso de activación (aHSC) que las estimula a sintetizar una gran cantidad de proteínas fibrogénicas (colágena I, III y VI), así como también hay una gran liberación de factores de crecimiento celular que inducen a las aHSC para que proliferen activamente. Todos estos procesos ocasionan que haya un gran depósito fibrilar en las fenestraciones endoteliales (capilarización del sinusoide) que impide el libre intercambio de moléculas entre el plasma sanguíneo y los hepatocitos, perdiéndose así las funciones vitales del órgano.

A pesar de los avances logrados en la última década en la comprensión de la cirrosis hepática, no existe cura farmacológica definitiva y sólo se dispone de tratamientos que contribuyen a aminorar los síntomas. En los últimos años están siendo exploradas nuevas drogas con resultados contradictorios. Sólo el trasplante de hígado ha logrado mejorar las condiciones de vida de estos enfermos. Sin embargo, las posibilidades de este procedimiento sofisticado y su alto costo son extremadamente limitadas para este grave problema de salud pública, por lo que continúa la búsqueda de combinaciones farmacológicas que resulten más eficaces y de nuevos fármacos sobre todo de aquellos que han probado 
su efectividad en procesos fibróticos experimentales que afectan a otros órganos.

\section{CÉLULAS FIBROGÉNICAS DEL HÍGADO}

Una gran cantidad de datos indican que la acumulación de MEC durante la fibrosis hepática se origina de dos tipos particulares de células miofibroblásticas que expresan actina $\alpha$ de músculo liso ( $\alpha$-SMA) derivadas de distintas poblaciones celulares, conocidas como HSCa y miofibroblastos hepáticos ${ }^{6}$.

Células estelares hepáticas (HSC). Comprenden de 5\%-10\% de las células en un hígado normal y se localizan en el espacio subendotelial (espacio de Disse) entre los hepatocitos y las células del endotelio sinusoidal (Figura 2). Estas células almacenan $80 \%$ de los retinoides (vitamina A) de la economía corporal en forma de retinol palmitato en gotas lipídicas dentro de su citoplasma. En condiciones fisiológicas normales, las HSC participan de manera importante en la regulación de la homeostasis de retinoides. Seguido del daño hepático crónico o agudo, las HSC sufren cambios fenotípicos que las inducen a perder sus retinoides y a sintetizar una gran cantidad de MEC (HSCa), este cambio es de tipo miofibroblástico. Dicha célula muestra propiedades fibrogénicas de novo, incluyendo proliferación y acumulación en las áreas necróticas de células parenquimales, secreción de citocinas proinflamatorias y quimiocinas ${ }^{6,7}$.

Miofibroblastos hepáticos. Son otro tipo de células fibrogénicas que derivan de fibroblastos de tejido conectivo portal, fibroblastos perivasculares de las venas centrales y portales y fibroblastos periductales en los tractos cercanos a las células del epitelio de los ductos biliares. Su participación en fibrogénesis se observó inicialmente en cirrosis biliar experimental al demostrar la transformación miofibroblástica de los fibroblastos periductales y portales precedida de la activación de las HSC en el lóbulo. Las propiedades funcionales y fenotípicas de los miofibroblastos hepáticos son similares a las HSCa. Estudios en cultivo han establecido claramente que algunos marcadores fenotípicos distinguen ambos tipos celulares, incluyendo la expresión selectiva de fibulina 2 e interleucina 6
(IL-6) por miofibroblastos hepáticos y la proteasa P100 y relina por HSCa. Respecto a las funciones biológicas, las HSCa muestran menores diferencias funcionales con los miofibroblastos hepáticos, tales como una vida corta que finalmente entra en apoptosis y baja capacidad proliferativa. Pero en trabajos futuros se necesita delinear la contribución precisa de cada tipo celular en el proceso fibrogénico y caracterización del linaje celular para proveer una información útil 6,8,9.

En la actualidad, las investigaciones concemientes a la búsqueda del agente celular causal de la cirrosis hepática han dado indicios de otra población migrante originada de médula ósea y que se conoce como fibrocitos, los cuales bajo algún estímulo fibrogénico por algún factor o citocina en un órgano las activan para que éstas abandonen la médula ósea y se dirijan a la región del daño ${ }^{4}$.

\section{MODELOS EXPERIMENTALES}

El desarrollo de drogas antifibróticas requiere la disponibilidad de sistemas experimentales apropiados para estudios preclínicos y para la definición de exámenes clínicos finales que garanticen la información recabada en los mismos.

Modelos de cultivo celular. Se han utilizado cultivos de HSC de humano y ratón y de miofibroblastos hepáticos de manera rutinaria para definir blancos antifibróticos y ensayar potenciales drogas contra la fibrosis. El aislamiento de HSC se basa en la digestión enzimática de hígados nomales y purificación de células con vitamina $\mathrm{A}$ a través de un gradiente de densidad. Durante pocos días, las HSC nicas en vitamina A adquieren características miofibroblásticas. Sin embargo, los fibroblastos hepáticos son obtenidos de explantes de hígado normal y no realizan una transformación fenotípica. Ambos tipos de células (HSC y fibroblastos) exhiben propiedades similares a las células fibrogénicas in vivo. Otros modelos de cultivo incluyen líneas de HSC de humano o ratón con características miofibroblásticas obtenidos espontáneamente o por transfección de la región codificante del virus SV-40. Pero la semejanza in vivo de estos modelos es cuestionable hasta el momento ${ }^{6}$.

Modelos animales. Estos se han utilizado de manera importante debido a su conveniente es- 
tructura de tiempo. Las características del proceso fibrogénico dependen de la naturaleza del daño. Componentes tales como tetracloruro de carbono $\left(\mathrm{CCl}_{4}\right)$, dimetilnitrosamina o galactosamina generan una significativa necrosis de hepatocitos asociada con una marcada inflamación y fibrosis. En estos modelos, el efecto antifibrótico de ciertas drogas puede también resultar en un efecto directo en las células fibrogénicas o de una actividad antiinflamatoria no específica. Otros modelos adicionales con baja inflamación son el de ligadura del conducto biliar y el de administración de tioacetamida, que pueden utilizarse en paralelo para validar la eficiencia de ciertas moléculas antifibróticas. Sin embargo, el daño hepático inducido en estos modelos puede ser revertido suspendiendo la administración de la hepatotoxina $\left(\mathrm{CCl}_{4}\right)$ o con una anastomosis bilio-digestiva en el caso del modelo de ligadura del ducto biliar ${ }^{6}$.

Ensayos de fibrosis en humanos. Durante años, la biopsia hepática ha sido el estándar de oro para monitorear la fibrosis en estudios clínicos. Existen algunos índices semi-cuantitativos, tales como el ampliamente utilizado índice de Knodell o el índice de Metavir. Sin embargo, la invasibilidad de la biopsia hepática limita las repeticiones seriadas del procedimiento. La cuantificación del área fibrótica por morfometría demuestra gran seguridad pero presenta un significativo coeficiente de variación. Finalmente el error de muestreo relacionado a la distribución heterogénea de la fibrosis ocurre en $15 \%$ a $25 \%$ de los casos, particularmente en estados avanzados. Estas limitaciones han estimulado la búsqueda de marcadores sénicos sensibles de fibrosis que no sean invasivos. Fragmentos de los constituyentes de la MEC (ensayo de hidroxiprolina) liberados en circulación durante la remodelación no han sido muy útiles, haciendo inadecuado el diagnóstico específico, particularmente para estadios de fibrosis intermedia. Recientes esfuerzos enfocados en índices con la utilización de combinaciones de marcadores proteicos de la MEC o basados en parámetros bioquímicos y hematológicos y más recientemente en análisis de glómico sérico han resultado satisfactorios. En este campo en expansión, el fibrotest combina cinco variables bioquímicas comunes. Finalmente, mediciones de elastometría hepática también muestra resultados promisorios que están siendo ensayados para validar exámenes multicéntricos ${ }^{6}$.

\section{ESTRATEGIAS ANTIFIBRÓTICAS}

Una droga antifibrótica ideal y específica del hígado debe evadir los efectos adversos sobre las proteínas de matriz extrahepática y debe selectivamente atenuar la excesiva deposición de colágena sin afectar la síntesis de MEC normal. Recientemente, la inhibición del daño parenquimal y la inflamación hepática han sido de gran interés.

Inhibición del daño al parénquima. Durante el daño hepático crónico, los hepatocitos y las células del epitelio biliar sufren un proceso de muerte apoptótica. Interesantemente, existe una asociación directa entre la apoptosis de hepatocitos y fibrogénesis hepática en algunos modelos experimentales. Por ejemplo, ratones con una linfoproliferación deficientes de Fas mostraron un decremento en la inflamación y fibrosis en un modelo de ligadura del ducto biliar. De manera similar, la fibrosis hepática inducida de manera inmunológica por repetidas administraciones de Concavalina A es fuertemente reducida por interferencia de ARN dependiente de Fas. Estos datos sugieren que la inhibición de apoptosis en hepatocitos e inflamación son interesantes para la prevención del proceso fibrótico. Respecto a este concepto, se ha demostrado en un estudio clínico de fase II que un inhibidor general de caspas (IDN-6556) redujo la apoptosis de hepatocitos y fibrosis en un modelo de ligadura del conducto biliar, pero esta estrategia que interviene en la vía apoptótica de hepatocitos presenta un alto riesgo de carcinogénesis a largo tiempo ${ }^{6}$.

Reducción de inflamación. La inflamación es comúnmente asociada con la progresión de la fibrosis hepática durante la enfermedad hepática crónica. Productos derivados de células de Kupffer y leucocitos estimulan las propiedades fibrogénicas de las HSC y miofibroblastos hepáticos. A este respecto, se han observado efectos benéficos con inductores de apoptosis de células Kupffer, tales como los inhibidores de la vía de la 5-lipoxigenasa, el cual reduce la inflamación y fibrosis hepática en el modelo de $\mathrm{CCl}_{4}{ }^{10}$. También se ha investigado el papel de la interleucina 10 (IL-10) en referencia a su papel positivo sobre la respuesta Th1 proinflamatoria en 24 pacientes con hepatitis C crónica, donde se demostró una sorprendente disminución 
de la inflamación que se asoció con un decremento en la fibrosis ${ }^{11}$.

\section{BLANCOS TERAPÉUTICOS}

Los blancos terapéuticos para fibrosis hepática se muestran en la Figura 3, e incluyen: remoción del agente lesivo, útil en pacientes que cursan con obstrucción biliar, en pacientes consumidores crónicos de bebidas alcohólicas y por virus de la hepatitis $\mathrm{B}$ y $\mathrm{C}$, siendo esta última la manera más difícil para inducir una regresión fibrótica, ya que se debe eliminar por completo el agente causal ${ }^{12-16}$.

El empleo de drogas con propiedades antioxidantes como vitamina $\mathrm{E}^{17,18}$ silimarina ${ }^{11}$, fosfatidilcolina ${ }^{19}$, S-adenosil-L-metionina, retinoides, resveratrol y quercetín ${ }^{20,21}$, en diferentes modelos experimentales mostraron que suprimen la respuesta fibrótica al daño oxidativo, sin embargo faltan estudios que valoren su seguridad y eficacia.

Para suprimir la respuesta inflamatoria se han utilizado los glucocorticoides pero no han logrado la supresión de la fibrogénesis y la progresión a la cirrosis continúa 22 . En un estudio retrospectivo, Yoneyama y cols, realizado en 29 pacientes con hepatitis autoinmune, se observó la aparición de algunos eventos adversos severos, como diabetes mellitus y algunos síntomas circulatorios y neurológicos $^{23-25}$. Con este mismo propósito se han probado la IL-10 e Inhibidores del factor de necrosis tumoral $\alpha$ (TNF- $\alpha$ ), con el objetivo de neutralizar citocinas proinflamatorias y lograr disminuir la necrosis y la inflamación en el hígado, pero faltan estudios para demostrar su efectividad ${ }^{11,26,27}$. El malotilate es un agente antiinflamatorio en proceso

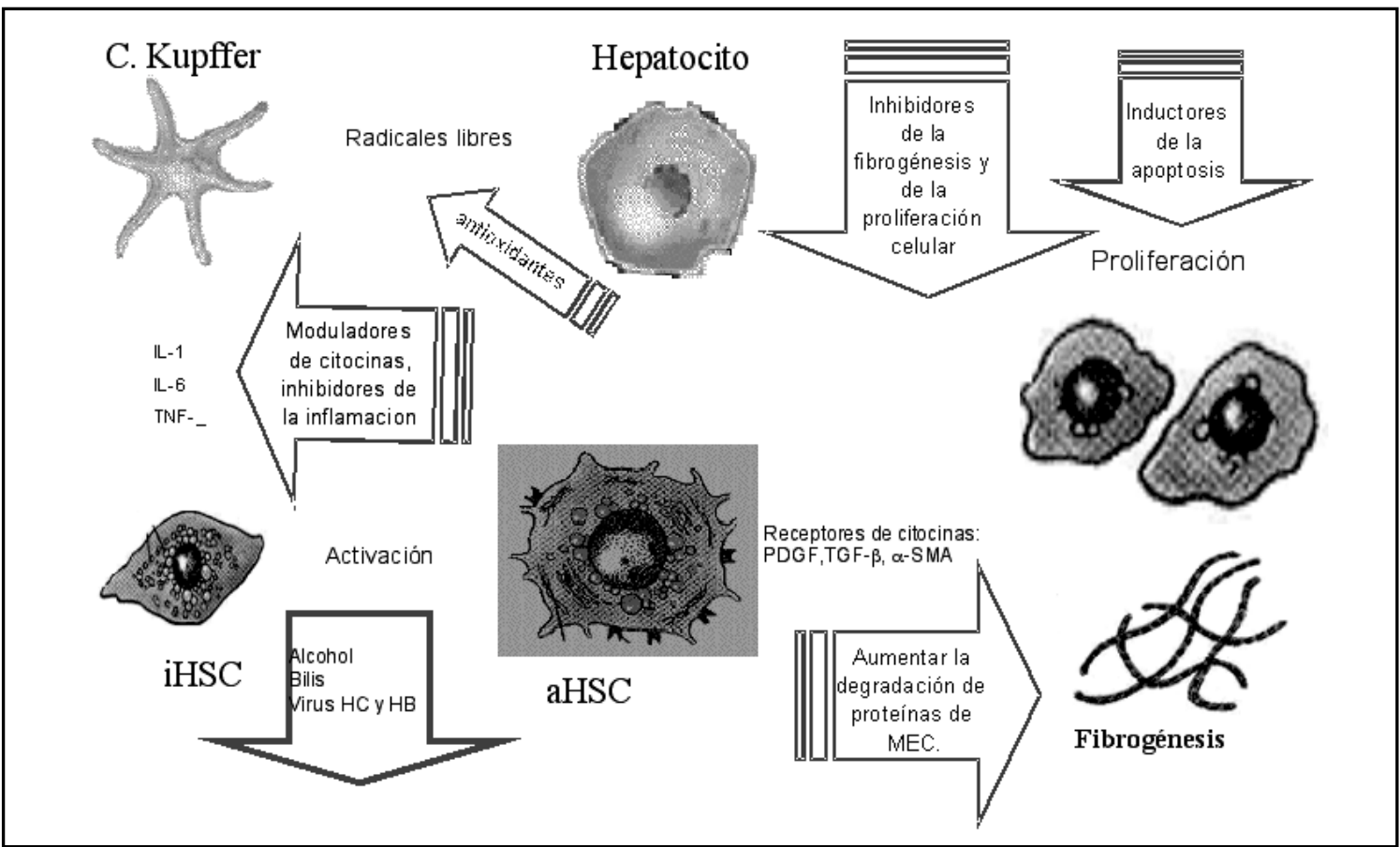

Figura 3. Eventos moleculares que conducen a la cirrosis hepática y que deben ser corregidos para el tratamiento de la enfermedad. Las flechas señalan las estrategias terapéuticas e incluyen: supresión el agente lesivo, reducción del estrés oxidativo, suprimir la respuesta inflamatoria, modular las citocinas proactivadoras de las células estelares hepáticas (HSC), modular el depósito de matriz extracelular y drogas que induzcan la apoptosis de las células estelares hepáticas activadas. IL-1; interleucina 1, IL-6; interleucina 6, TNF- $\alpha$; factor $\alpha$ de necrosis tumoral, iHSC; célula estelar hepática inactiva; aHSC; célula estela hepática activa, PDGF; factor de crecimiento derivado de plaquetas, TGF- $ß$; factor $\beta$ de crecimiento transformante, $\alpha$-SMA; $\alpha$ actina de músculo liso, MEC; matriz extracelular. 
de investigación en fibrosis hepática, con efecto hepatoprotector en modelos animales por su capacidad de inhibir la actividad del citocromo P-450 y reducir el depósito de colágena. Sin embargo, esta droga no mejora la sobrevida ni la arquitectura histológica de pacientes con cirrosis biliar primaria y cirrosis hepática alcohólica ${ }^{28}$. De la medicina tradicional china el sho-saiko-to demostró en diferentes modelos experimentales la capacidad de inhibir la activación y proliferación de las HSC reduciendo el estrés oxidativo, y el depósito de colágena ${ }^{29,30}$, de igual forma glicirrizina, un extracto de orozuz atenúa la fibrosis en ratas inducida por ligadura del ducto biliar, al parecer por sus propiedades antioxidantes y por su capacidad de inhibir la activación del factor nuclear $\mathrm{kB}$ (NFkB). Interferón (IFN) $\alpha$ y $\gamma$ mostraron que inhiben la activación y el crecimiento de las HSC suprimiendo la síntesis de colágena y fibronectina en más de $40 \%$ con respecto al control.

Otras estrategias se han desarrollado para inducir o bloquear el papel de citocinas involucradas en el proceso fibrogénico y de proliferación celular, en este sentido el HGF se ha utilizado en estudios experimentales que demostraron un importante papel para inhibir la activación de las HSC. El refanlin por su capacidad de mimetizar la actividad in vitro del HGF, se está probando en cirrosis hepática experimental con la ventaja de que está disponible para su administración oral. Otras drogas que se han desarrollado, para inhibir citocinas involucradas en el proceso de iniciación y perpetuación del evento fibrogénico del hígado, incluyen el mesilato de camostat, un inhibidor de la proteasa de serina, que inhibe la liberación de TGF-ß1 de su proteína de unión, y el receptor tipo II dominante negativo de TGF-ß1, que pretende antagonizar el efecto del TGF- $ß 1$ e impide la cascada de señalización intracelular para que no se expresen genes profibrogénicos como el gen de colágena ${ }^{31,32}$.

Existen varios blancos terapéuticos para controlar los depósitos elevados de colágena ya que su producción se encuentra regulada a diferentes niveles: transcripcional, postranscripcional y traduccional, al igual que su degradación por MMPs, como los inhibidores de la prolil-4 hidroxilasa (el HOE077 y el S 4682) que demostraron una gran capacidad para inhibir la fibrosis en modelos experimentales ${ }^{33-36}$. La halofunginona un com- puesto antimicrobiano con potente efecto inhibidor de la síntesis de colágena, también mostró eficacia en modelos de fibrosis renal ${ }^{37}$ y en cirrosis hepática en ratas inducida por dimetilnitrosamina ${ }^{38}$.

La colchicina, un agente que bloquea la polimerización de tubulina demostró que inhibe la secreción de colágena y que aumenta la actividad de colagenasa in vitro ${ }^{39,40}$. Además, la administración oral de esta droga disminuyó el desarrollo de fibrosis en ratas intoxicadas con $\mathrm{CCl}_{4}{ }^{41}$. La colchicina se ha usado en algunos ensayos clínicos y ha mostrado cierto efecto benéfico en la cirrosis de varias etiologías ${ }^{42}$. Sin embargo, estos resultados no fueron satisfactorios en pacientes con cirrosis biliar primaria ${ }^{43-45}$. Estas evidencias muestran que la colchicina es ineficaz para frenar la progresión de la cirrosis hepática.

También se han probado substancias que inhiben las vías de señalización de algunas moléculas profibrogénicas, como la pentoxifilina; un inhibidor de la enzima fosfodiesterasa, que disminuye la proliferación de las HSC in vitro e in vivo y previene las señales relacionadas con el PDGF que participa en el control mitogénico de las HSC $^{46-49}$. De igual forma se realizó un experimento para bloquear esta citocina mediante una estrategia antisentido sobre la cadena $§$ del PDGF en donde se obtuvieron resultados prometedores $^{50}$. El ácido S-farnesiltiosalicílico; un antagonista de ras inhibe la proliferación y migración de las HSC y reduce la fibrosis en ratas inducida por tioacetamida ${ }^{51}$. Los antagonistas del receptor de angiotensina 1 (AT-1); que son útiles para tratar la fibrosis renal y cardíaca, han mostrado que reducen la fibrosis hepática en ratas ligadas del conducto biliar ${ }^{52}$.

\section{VANGUARDIA EN INVESTIGACIÓN TERAPÉUTICA}

También en estos últimos años se ha aumentado el empleo de nuevos fármacos que han sido útiles en otras formas de fibrosis que afectan a otros órganos. A este respecto hacia 1994 se dio a conocer un reporte preliminar donde publican las propiedades antifibróticas de pirfenidone ${ }^{53}$, una nueva droga inicialmente conocida sólo por su capacidades analgésicas y antiinflamatorias y que ha sido capaz de prevenir el desarrollo de lesiones 
fibróticas y de resolver las lesiones ya existentes en el modelo de fibrosis pulmonar inducida en hámster y recientemente gran número de publicaciones sobre estudios in vitro e in vivo reportan la utilidad de pirfenidone en fibrosis pulmonar y de otros órganos incluida la cirrosis hepática ${ }^{54}$.

En cirrosis hepática experimental, recientemente se ha avanzado con el empleo de la terapia génica, con el fin de aumentar la actividad de las MMPs que degradan el excesivo acúmulo de proteínas fibrilares (principalmente colágenas). Al respecto se ha reportado el empleo de un vector adenoviral con el activador de plasminógeno tipo urocinasa humano (Ad-huPA), el cual es un iniciador de la cascada proteolítica, con la finalidad de revertir la fibrosis hepática experimental inducida en ratas, con resultados alentadores ${ }^{55,56}$. Otras estrategias de terapia génica que se encuentran en fase experimental en animales, incluyen el empleo de MMP-8 (Ad-MMP8) y MMP-157, tanto en el modelo de ligadura del ducto biliar como en el de $\mathrm{CCl}_{4}{ }^{58-60}$. Otra estrategia más es el bloqueo de la citocina profibrogénica más importante -TGFßen esta patología, a través de un receptor dominante negativo ( $\Delta$ CitTßRII) que ha sido modificado de tal manera que no posea la región citoplásmica bloqueando así la cascada de señalización intracelular e impidiendo que lleve a cabo

\section{REFERENCIAS}

1. Gressner A, Bachem M. Cellular sources of noncollagenous matrix proteins: role of fat-storing cells in fibrogenesis. Semin Liver Dis 1990; 10: 30-46.

2. GRESSNER A, BACHEM M. Molecular mechanism of liver fibrogenesis: a homage to the role of activated fat-storing cells. Digestion 1995; 56: 335-46.

3. Senties-Gómez D, Gálvez-Gastelum Fu, Armendárz-BoRUNDA J. Fibrosis hepática: el papel de las metaloproteinasas y TGF-B. Gac Med Mex 2005; 141: 307-14.

4. BATALER R, BRENNER DA. Liver fibrosis. J Clin Invest 2005; 115: 209-18.

5. Olaso E, Friedman S. Molecular regulation of hepatic fibrogenesis. J Hepatol 1998; 29: 836-47.

6. Lotersztajn S, Julien B, Teix-Clerc F, Grenard P, MaLat A. Hepatic fibrosis: molecular mechanism and drug targets. Annu Rev Pharmacol Toxicol 2005; 45: 605-28. sus efectos fibrogénicos ${ }^{31}$. Finalmente se está trabajando con una combinación terapéutica de Ad-MMP8 y Ad-huPA en la reversión de la fibrosis y sus efectos benéficos sobre la encefalopatía hepática experimental ${ }^{61}$.

\section{CONCLUSIONES}

Existe una gran cantidad de información respecto a los mecanismos fisiopatológicos que participan durante el desarrollo de la fibrosis hepática, tal parece que esta patología se desencadena de manera multifactorial debido a la gran variedad de mecanismos moleculares y celulares que participan. Sin embargo, con este advenimiento en el conocimiento de la cirrosis hepática, también se han desarrollado un sinnúmero de estrategias terapéuticas para tratar de detener el avance, disminuir o revertir el proceso fibrótico, pero tal parece que aún la ciencia no ha dado en el blanco para curar por completo la enfermedad. Recientemente se están desarmollando estrategias moleculares (como la terapia génica) con resultados prometedores y más aún se están desarrollando estrategias moleculares farmacológicas combinadas para no sólo atacar una posible ruta alterada durante la patología, sino de una manera multifactorial.

7. SENOO H. Structure and function of hepatic stellate cells. Med Electron Microsc 2004; 37: 3-15.

8. Forbes S, Russo FP, Rey V, Burra P, Rugge M, Wright NA ET AL. A significant proportion of myofibroblast are bone marrow origin in human liver fibrosis. Gastroenterology 2004; 126: 955-64.

9. BABASA S. Commitment of bone marrow cells to hepatic stellate cells in mouse. J Hepatol 2004; 40: 255-60.

10. Tiтоs E. Inhibition of 5-lipoxygenase induces cell growth arrest and apoptosis in ral kupffer cells: implications for liver fibrosis. FASEB J 2003; 17: 1745-47.

11. Nelson D, Lauwers G, Iau J. Interleukin 10 treatment reduces fibrosis in patients with chronic hepatitis C: a pilot trial of interferon nonrespponders. Gastroenterology 2000; 118: 655-60.

12. Shiratori Y, Imazeki F, Moriyama M. Histologic improvement of fibrosis in patients with hepatitis 
C who have sustained response to interferon therapy. Ann Intern Med 2000; 132: 517-24.

13. Hammel P, Couvelard A, O'Toole D. Regression of liver fibrosis after biliary drainage in patients with chronic pancreatitis and stenosis of the common bile duc. N Engl J Med 2001; 344: 418-23.

14. Pares A, Cabalieria J, Bruguera M. Histological course of alcoholic hepatitis. Influence of abstinence, sex and extent of hepatic damage. J Hepatol 1986; 2: 3-42.

15. Ramm G, Crawford D, Powell L Hepatic stellate cell activation in genetic haemochromatosis. Lobular distribution, effect of increasing hepatic iron and response to phlebotomy. J Hepatol 1997; 26: 584-92.

16. SchilsKy M, Scheinberg I, SternLeb I. Prognosis of wilsonian chronic active hepatitis. Gastroenterology 1991; 100: 762-7.

17. Parola M, Leonarduzzi G, Biasi F. Vitamin E dietary supplementation protects against carbon tetrachloride-induced chronic liver damage and cirrhosis. Hepatology 1992; 16: 1014-21.

18. De La Maza M. Effects of long-term vitamin E supplementation in alcoholic cirrhotics. J Am Coll Nutr 1995; 14: 192-6.

19. MA X, ZHAO J, LEBER C. Polyenylphosphatidylcholine attenuates non-alcoholic hepatic fibrosis and accelerates its regresión. J Hepatol 1996; 24: 604-13.

20. Mizobushi Y, Shimizu I, Yasuda M. Retinyl palmitate reduces hepatic fibrosis in rats induced by dimethylnitrosamine or pig serum. J Hepatol 1998; 29: 933-43.

21. Godichaud S, Krisa S, Couronne B. Deactivation of cultured human liver myofibroblast by transresveratrol, a grapevine-derived polyphenol. $\mathrm{He}$ patology 2000; 31: 922-31.

22. Summerskill W, Korman M, Ammon H. Prednisone for chronic active liver disease: dose tritration, standart dose, and combination with azathioprine compared. Gut 1975; 16: 876-83.

23. Czaja A, Ammon H, Summeskil W. Clinical features and prognosis of severe chronic active liver disease (CALD) after corticosteroid-induced remission. Gastroenterology 1980; 78: 518-23.

24. Davis G, Czaja A, Ludwing J. Development and prognosis of histologic cirrosis in corticosteroidtreated hepatitis B surface antigen-negative chronic active hepatitis. Gastroenterology 1984; 87: 1222-27.

25. YoNEYAMA K. Efficacy and safety of prednisolone in patients with autoimmune hepatitis. Adv Ther 2006; 23: 74-91.
26. Manzini R, Benedetti A, Jezequel A. An interleukin1 receptor antagonist decreases fibrosis induced by dimethylnitrosamine in rat liver. Virchows Arch 1994; 424: 25-31.

27. Bruck R, Hershroviz R, Lider O. The use of synthetic analogues of Arg-Gly-Asp (RGD) and soluble receptor of tumor necrosis factor to prevent acute and chronic experimental liver injury. Yale J Biol Med 1997; 70: 391-402.

28. Ryhanen L, Stendack F, Ala-Koкko L. The effect of malotilate on type III and type IV collagen, laminin and fibronectin metabolism in dimethylnitrosamine-induced liver fibrosis in the rat. J Hepatol 1996; 24: 238-45.

29. Shimizu I, Ma Y, Mizoвuchi Y. Effects of Sho-saikoto, a Japanese herbal medicine, on hepatic fibrosis in rats. Hepatology 1999; 29: 149-60.

30. Mryamura M, Ono M, Kyotani S. Effects of sho-saikoto extracts on fibrosis and regeneration of the liver in rats. J Pharm Pharmacol 1998; 50: 97-105.

31. Hernández-CaÑaveral I, González J, López-CASilias F, ARMEndaRIZ-Borunda J. Amplified expression of dominant-negative transforming growth fatctorbeta type II receptor inhibits collagen type I production via reduced Smad-3 activity. J Gastroenterol Hepatol 2004; 19: 380-7.

32. Gálvez-Gastelum Fu, Sandoval-Rodríguez A, ArmenDÁRIZ-BoRUNDA J. El factor de crecimiento transformante $\beta$ como blanco terapéutico. Sal Pub Mex 2004; 46: 341-50.

33. Sakaida I, Uchida K, Hirokana K. Prolyl 4-hidroxylase inhibitor (HOE 077) prevents TIMP-1 gene expresión in rat liver fibrosis. J Gastroenterol 1999; 34: 376-7.

34. Sakaida I, Matsumura Y, Kubota M. The prolilbb 4hidroxylase inhibitor HOE 077 prevents activation of Ito cells, reducing procollagen gene expresión in rat liver fibrosis induced by cholinedeficient L-amino acid-defined diet. Hepatology 1996; 23: 755-63.

35. Bickel M, Baringhaus K, GerL M. Selective inhibition of hepatic collagen accumulation in experimental liver fibrosis in rats by a new prolyl 4-hidrocylase inhibitor. Hepatology 1998; 28: 404-11.

36. WANG Y, WANG S, BICKEL M. Two novel antifibrotics, HOE 077 and Safironil, modulate stellate cell activation in rat liver injury: differential effects in males and females. Am J Pathol 1998; 152: 279-87.

37. Nagier A, Gofrit O, OHANa M. The effect of halofuginone, an inhibitor of collagen type I synthe- 
sis, on urethral stricture formation: in vivo and in vitro study in a rat model. J Urol 2000; 164: 1776-80.

38. Pines M, Knopov V, Genina O. Halofuginone, a specific inhibitor of collagen type I synthesis, prevents dimethylnitrosamine-induced liver cirrosis. J Hepatol 1997; 27: 391-8.

39. HaRRIS E, KRANE S. Effects of colchicines on collagenase in cultures of rheumatoid synovium. Arthritis Rheum 1971; 14: 669-84.

40. Diegelmann R, Peterkoffsky B. Inhibition of collagen secretion from bone and culture fibroblasts by microtubular disruptive drugs. Proc Natl Acad Sci 1972; 69: 892-6.

41. RojKind M, KeRSHENobich D. Effect of colchicine on collagen, albumin and transferrin sintesis by cirrhotic rat liver slices. Biochim Biophys Acta 1975; 378: 415-23.

42. Kershenobich D, Vargas F, García-Tsao G. Colchicina in the treatment of cirrosis of the liver. N Engl J Med 1988; 318: 1709-13.

43. Bodenheimer H, Schaffner F, Pezzulo J. Evaluation of colchicine therapy in primary biliary cirrosis. Gastroenterology 1988; 95: 124-9.

44. Kaplan M, Awing D, ZimMERMan H. A prospective trial of colchicine for primary biliary cirrosis. N Engl J Med 1986; 315: 1448-54.

45. WARnes T, SMith A, LEE F. A controlled trial of colchicines in primary biliary cirrhosis. Trial design and preliminary report. J Hepatol 1987; 5: 1-7.

46. Preaux A, Mallat A, Rosenbaum J. Pentoxifylline inhibits growth and collagen synthesis of cultured human hepatic myofibroblast-like cells. Hepatology 1997; 26: 315-22.

47. Pinzani M, Marra F, Callgiuri A. Inhibition by pentoxifylline of extra-cellular signal-regulated kinase activation by platelet-derived growth factor in hepatic stellate cells. Br J Pharmacol 1996; 119: 1117-24.

48. WindMEIER C, GRESSNER A. Pharmacological aspects of pentoxifylline with emphasis on its inhibitory actions on hepatic fibrogenesis. Gen Pharmacol 1997; 29: 181-96.

49. Sveglati-Baroni G, Di Sario A, Casini A. The Nat/ $\mathrm{H}+$ exchanger modulates the fibrogenic effect of oxidative stress in rat hapatic stellate cells. J Hepatol 1999; 30: 868-75.

50. Borkham-Kamphrost E, Sto D, Gressner A, WeiskirCHEN R. Antisense strategy against PDGF B-chain proves effective in preventing experimental liver fibrosis. BBRC 2004; 321: 413-23.

51. ReIF S, Weis B, AeEd H. The Ras antagonist, farnesylthiosalicylic acid (FTS), inhibits experimentally-induced liver cirrosis in rats. J Hepatol 1999; 31: 1053-61.

52. Jonson J, Clouston A, Ando Y, Keiemer L, Horn M, Adamson M ET aL. Angiotensin converting enzyme inhibition attenuates the development of hepatic fibrosis in the rat bile duct ligation model. Hepatology 2000; 32: 191.

53. Margoln S, LefKowitz S. Pirfenidone: a novel pharmacologic agent for prevention and resolution (removal) of lung fibrosis. FASEB J 1994; 8: A117.

54. García L, Hernández I, Sandoval A, Salazar A, García J, VERA J ET AL. Pirfenidone effectively reverses expenmental liver fibrosis. J Hepatol 2002; 37: 797-805.

55. Miranda-Díaz A, Rincon AR, Salgado S, Vera-Cruz J, Gálvez J, Islas MC et al. Improved effects of viral gene delivery of human uPA plus biliodigestive anastomosis induce recovery from experimental biliary cirrhosis. Mol Ther 2004; 9: 30-7.

56. Salgado S, García J, Vera J, Siluer F, Bueno M, Miranda A ET aL. Liver cirrosis is reverted by urokinase-type plasminogen activator gene therapy. Mol Ther 2000; 2: 545-51.

57. Iimuro Y, Nishio T, Morimoto T, NitTa T, Stefanovic B, Сно SK ET AL. Delivery of matriz metalloproteinase-1 attenuates established liver fibrosis in the rat. J Clin Invest 2001.

58. Siluer-López F, Sandoval A, Salgado S, Salazar A, Bueno M, García J et al. Treatment with human metalloproteinase-8 gene delivery ameliorates experimental rat liver cirhosis. Gastroenterology 2004; 126: 1122-33.

59. García-Bañuelos J, Siluer-López F, Miranda A, Aguiiar LK, Aguilar-Córdova E, Armendariz-Borunda J. Cirrhotic rat livers with extensive fibrosis can be safety transduced with clinical grade adenoviral vectors. evidence of cirrhosis reversion. Gen Ther 2002; 9: 127-34.

60. Prosser C, Yen RD, Wu J. Molecular therapy for hepatic injury and fibrosis: where are we? World J Gastroenterol 2006; 12: 509-15.

61. Gálvez-Gastelum Fu, Segura A, García J, Beas C, Armendariz-Borunda J. Combined HUPA plus MMP8 gene therapy reverts cirhosis and improves hepatic encephalopathy. Gut 2004; 53: A259-A260. 